(C) Cambridge University Press 2009. The online version of this article is published within an Open Access environment subject to the conditions of the Creative Commons Attribution-NonCommercial-ShareAlike

licence <http://creativecommons.org/licenses/by-nc-sa/2.5/>. The written permission of

Cambridge University Press must be obtained for commercial re-use.

\title{
Differential predictions about future negative events in seasonal and non-seasonal depression
}

\author{
T. Dalgleish ${ }^{1 *}$, A.-M. J. Golden ${ }^{1}$, J. Yiend ${ }^{2}$ and B. D. Dunn ${ }^{1}$ \\ ${ }^{1}$ Medical Research Council, Cognition and Brain Sciences Unit, Cambridge, UK \\ ${ }^{2}$ Institute of Psychiatry, University of London, London, UK
}

Background. Previous research indicates that individuals with seasonal depression (SD) do not exhibit the memory biases for negative self-referent information that characterize non-seasonal depression (NSD). The current study extended this work by examining processing of self-referent emotional information concerning potential future events in SD

Method. SD and NSD patients, along with never-depressed controls, completed a scenario-based measure of likelihood estimation for future positive and negative events happening either to the self or to another person.

Results. SD patients estimated future negative events as more likely to happen to both the self and others, relative to controls. In contrast, in the NSD sample this bias was specific to self-referred material. There were no group differences for positive events.

Conclusions. These data provide further evidence that the self-referent bias for processing negative information that characterizes NSD can be absent in SD, this time in the domain of future event processing.

Received 29 July 2008; Revised 29 May 2009; Accepted 11 June 2009; First published online 23 July 2009

Key words: Major depressive disorder, prospective cognition, seasonal affective disorder, seasonal depression.

\section{Introduction}

Seasonal depression (SD), also known as seasonal affective disorder, is a category of major depressive disorder (MDD) where the depressive episodes follow a clear seasonal pattern. The predominant presentation is a major depressive episode (MDE) in the autumn and winter months with remission in the spring and summer (Rosenthal et al. 1984). Such winter SD has many symptoms in common with nonseasonal MDD (NSD), e.g. depressed mood, loss of interest, negative thoughts and feelings about the self, anhedonia and suicidal ideation. However, winter SD is characterized by reversed or atypical vegetative symptoms including increased appetite (especially craving for carbohydrates), hyperphagia, hypersomnia, energy loss and weight gain. SD has an approximate $5 \%$ prevalence rate in the USA, with the majority of sufferers being female (Diagnostic and Statistical Manual of Mental Disorders, 4th edn, text

* Address for correspondence: Dr T. Dalgleish, MRC Cognition and Brain Sciences Unit, 15 Chaucer Road, Cambridge CB2 7EF, UK.

(Email : tim.dalgleish@mrc-cbu.cam.ac.uk) revision; DSM-IV-TR). SD is thought to result from disturbances in the body's circadian rhythms as a result of seasonal changes in daylight duration (Rosenthal \& Wehr, 1992). Consequently, research examining similarities and differences between SD and NSD has historically focused on these biological systems (Sohn \& Lam, 2005). However, more recent research efforts have targeted cognitive processing in SD and NSD.

\section{Cognitive processes in SD and NSD}

Research on cognitive processing in SD is notably scarce, compared with the enormous research database for NSD. However, a handful of studies indicate that SD is characterized by deficits in aspects of neuropsychological functioning that mirror those found in NSD, including compromised spatial recognition, short-term memory, learning, psychomotor speed, visual construction and self-reported cognitive failures (O'Brien et al. 1993; Michalon et al. 1997; Sullivan \& Payne, 2007).

Complementing these neuropsychological investigations, a small number of studies have focused on 
cognitive processing of emotionally negative information in SD. It is now well established that NSD is characterized by pervasive cognitive processing biases in favour of negative self-referent material (Williams et al. 1997). These biases impact upon perception (Weniger et al. 2004), attention (Mathews et al. 1996), memory (Blaney, 1986), attributions (Hammen et al. 1981), interpretations (Bisson \& Sears, 2007) and thinking (Beck, 1967; Beck et al. 1979), and are thought to play a key role in the onset and maintenance of NSD (Beck, 2005; Beck et al. 1979). There is now a growing body of evidence that similar biases are present in those with SD.

For example, SD patients present with depressotypic attributions in the same way as NSD individuals, such that negative events are perceived to have personal, global and stable causes, (Hodges \& Marks, 1998; Levitan et al. 1998; Dalgleish et al. 2004). Similarly, reported elevated levels of negative thinking, dysfunctional attitudes about the self and the world, and rumination on negative themes in SD samples (Hodges \& Marks, 1998; Rohan et al. 2003; Young \& Azam, 2003; Golden et al. 2006) are similar to those from studies of NSD patients (Hollon \& Kendall, 1980). Finally, selective attentional bias for negative material in SD (Spinks \& Dalgleish, 2001) is comparable with that found in NSD samples (Mathews et al. 1996).

All of these neuropsychological and cognitive bias studies discussed so far suggest that patients with SD present with a similar cognitive profile to those with NSD and are consequently consistent with arguments that SD and NSD are not necessarily distinct syndromes. In contrast, two recent studies suggest that the patterns of cognitive processing across the syndromes may not be isomorphic. In the domain of emotional word-list learning, NSD patients reliably show a mood-congruent memory bias in favour of recalling negative self-referent adjectives from mixed word lists that they have learned (Blaney, 1986). Intriguingly, this bias appears to be absent in NSD (Dalgleish et al. 2004). Similarly, in the domain of autobiographical recollection of emotional memories, the typical and reliable finding in NSD is of a relative difficulty in generating specific personal memories, located in time and place, when cued to do so. Instead, NSD patients are more likely to produce categorical descriptions of their autobiography that conflate across a number of past events in their lives (Williams et al. 2007). This relative 'over-generality' appears not to characterize SD participants who in fact tend to be more specific in their autobiographical recall than never-depressed controls (Dalgleish et al. 2001).

Taken together, these latter two studies indicate that patients with SD may not display the biased patterns of remembering negative self-referent information that typify NSD (Dalgleish et al. 2004). Given the proposed key role of self-referent processing of negative information in the onset, maintenance and treatment of NSD according to cognitive theorists (Beck et al. 1979; Beck, 2005), these findings could have important implications for the understanding and treatment of SD.

A pressing research objective is therefore to investigate other domains of self-referent processing of negative information in SD. To that end, the present study focuses on the processing of information concerning future self-referent events and experiences, which has also been shown repeatedly to be negatively biased in NSD (Butler \& Mathews, 1983; Andersen et al. 1992; Reich \& Weary, 1998).

\section{Negatively biased processing for future events}

A central domain of future event processing concerns the estimations of likelihood that people make about the occurrence of positive and negative experiences; for example, how likely it is that they will get cancer, or win a significant amount of money. Likelihood estimations are known to influence behavioural choices (Gregory et al. 1982) and so examining such estimations provides a useful window into the broader domain of prospective cognitive processing.

In a prototypical study of likelihood estimation (Butler \& Mathews, 1983), NSD and never-depressed participants estimated the likelihood of occurrence of a range of positive and negative events, in relation both to themselves and to other people, using a scenario-based measure. The results revealed no significant differences between the groups for positive events. However, NSD patients rated negative events as more likely to happen than did controls. Importantly, although controls did not differentiate between probability estimates for themselves and for others, NSD patients exhibited a self-referent bias in that ratings of the likelihood of negative events made for themselves were significantly higher than ratings of the same items when made for another person.

If the absence of the typical negative self-referent processing biases for past events in memory experiments in SD (Dalgleish et al. 2001, 2004) also applies to the processing of future events, then one would predict a different pattern of likelihood estimations for SD sufferers than for their NSD peers on this kind of likelihood estimation task (Butler \& Mathews, 1983). Specifically, the hypothesis would be that SD participants would not estimate negative events as more likely to happen to themselves, relative to other people (the negative self-referent bias) unlike the NSD patients in the original studies (Butler \& Mathews, 
1983). The primary aim of the present study was to address this question.

A significant limitation of the vast majority of research on cognitive processing in $\mathrm{SD}$ has been the absence of NSD comparison samples in the relevant studies. Indeed, we are aware of only two exceptions to this rule (Levitan et al. 1998; Sullivan \& Payne, 2007), both of which have actually revealed similar processing profiles across the two syndromes. Consequently, to date there are no studies to our knowledge that demonstrate reliable differences in cognitive processing between SD and NSD by directly comparing the two disorders within the same study. A key component of the present methodology was consequently the inclusion of SD, NSD and never-depressed samples within the same experimental protocol.

The present study therefore examined likelihood estimations for potential future positive and negative events with reference to the self and others in SD, NSD and never-depressed controls. Our specific hypothesis was that there would be a significant interaction for likelihood estimations of group, reference (self versus other) and event type (positive versus negative). Specifically, we predicted that the three groups would not differ in their estimations regarding positive events, whereas for negative events we expected the NSD group to show the standard self-referent bias, estimating such events as more likely to happen to the self than to another, but for there to be no support for this self-reference effect in either SD participants or in controls.

\section{Method}

\section{Study population}

Thirty-three participants with SD were recruited via advertisements placed with local self-help organizations and health centres. The principal criterion for inclusion in the SD group was meeting criteria for recurrent mood disorder with seasonal pattern (DSMIV-TR), currently in episode, as assessed during the winter season by the Structured Clinical Interview for DSM (SCID; First et al. 1997). It is important to note that a diagnosis of SD requires the existence of at least two prior MDEs with a reliable seasonal pattern. Seasonality was objectively validated in a subset of the present SD sample $(n=24)$ who were reassessed in the summer on the SCID, at which point all of them were in remission from depression and none met criteria for a hypomanic episode. In addition, SD group inclusion criteria were: no evidence of psychosis or organic brain damage; and being between the ages of 18 and 60 years. SD participants had a mean age of 44.42 (s.D. $=10.22)$ years. There were 25 women and eight men, similar to SD gender ratios described elsewhere (DSM-IV-TR).

Fourteen participants with non-seasonal recurrent MDD (the NSD group) were recruited via similar means. Criteria for inclusion in the NSD group were as for the SD group except that participants had to meet DSM-IV criteria for MDD with no seasonal pattern (APA, 1994), currently in episode according to the SCID, during the winter season. To ensure comparability with the SD group, NSD participants were also required to have had at least two prior MDEs. The NSD participants had a mean age of 51.50 (S.D. $=10.42$ ) years, with seven females.

Fifteen participants recruited from the volunteer panel of the Medical Research Council (MRC) Cognition and Brain Sciences Unit comprised the never-depressed control group. The inclusion criteria for the controls were the same as for the SD and NSD groups, with the exception that they did not meet criteria for current or past MDD, seasonal or nonseasonal, according to the SCID. The controls had a mean age of 45.60 (S.D. $=4.48$ ) years, with 10 females.

After complete description of the study to the participants, written informed consent was obtained. The study was approved by the University of Cambridge, Psychology Research Ethics Committee. The investigation was carried out in accordance with the Declaration of Helsinki.

\section{Materials}

\section{Mood measures}

The SD participants were administered the Structured Interview Guide for the Hamilton Depression Rating Scale, seasonal affective disorder version (SIGH-SAD; Williams et al. 1988) - a 29-item measure with established validity and reliability that assesses the symptoms of SD. All three groups also completed the Spielberger State Trait Anxiety Inventory (STAI; Spielberger et al. 1970) and the Beck Depression Inventory (BDI; Beck et al. 1961) - standard self-report measures of anxious and depressed mood, respectively, with well-established validity and reliability.

\section{Likelihood Estimation Measure (LEM) (Butler $\mathcal{E}$}

Mathews, 1983)

The LEM is an established scenario-based instrument designed to assess participants' estimations of the likelihood of a variety of potential future positive and negative events. The mini scenarios have high face validity and were selected to ensure that estimations of their likelihood were not subject to floor and ceiling effects. The LEM consists of 31 test scenarios and five practice scenarios. Of the 31 test scenarios, there are 
Table 1. Descriptive statistics for the SD, control and NSD groups

\begin{tabular}{lccl}
\hline & SD $(n=33)$ & $\begin{array}{l}\text { Control } \\
(n=15)\end{array}$ & NSD $(n=14)$ \\
\hline Age (years) & $42.14(12.23)$ & $45.60(4.49)$ & $51.50(10.42)$ \\
SIGH-SAD $^{\mathrm{a}}$ & $31.55(11.39)$ & & \\
BDI $^{\mathrm{b}}$ & $19.81(12.61)$ & $4.00(3.16)$ & $21.29(9.91)$ \\
STAI-state & $42.82(11.42)$ & $26.47(5.45)$ & $45.86(13.42)$ \\
STAI-trait $^{\mathrm{b}}$ & $53.34(11.37)$ & $33.87(6.90)$ & $61.36(5.01)$ \\
\hline
\end{tabular}

SD, Seasonal depression; NSD, non-seasonal depression; SIGH-SAD, Structured Interview Guide for the Hamilton Depression Rating Scale, seasonal affective disorder version; BDI, Beck Depression Inventory; STAI-state/trait, Spielberger State Trait Anxiety Inventory - state/trait scale.

Values are given as mean (standard deviation).

a Of the SD participants, 11 did not complete the SIGH-SAD.

${ }^{\mathrm{b}}$ Of the SD participants, one did not complete this measure.

seven fillers and 24 critical scenarios. These 24 scenarios consist of 12 pairs, six positive and six negative. One member of each pair is self-referent (e.g. 'What is the likelihood that, if you surprised a burglar in your home, he would attack you?'), while the other member is either left unspecified or is a specified individual with no obvious connection to the rater (e.g. 'What is the likelihood that the next person to fill in this questionnaire will get stomach cancer?'). The scenarios pertaining to self versus other are separated and randomly distributed within the measure to reduce strategic matching of responses. Responses reflect participants' estimated likelihoods that the given events will occur in the future. Responses consist of selections on a nine-point (0-8) scale ranging from 'not at all likely' to 'extremely likely'. Four scores are computed by summing across the relevant items reflecting self-referent negative events, self-referent positive events, other-referent negative events and other referent positive events. Each summary score ranges from 0 to 48 , with higher scores reflecting higher estimations of likelihood in that particular domain. The LEM showed good reliability in the present sample $(\alpha=0.81)$.

\section{Procedure}

Assessment was face to face on an individual basis. After completing informed consent forms, participants were administered the SCID followed by the LEM. The vast majority of the SD group were next assessed using the SIGH-SAD, before they all completed the BDI and STAI. The NSD group and controls

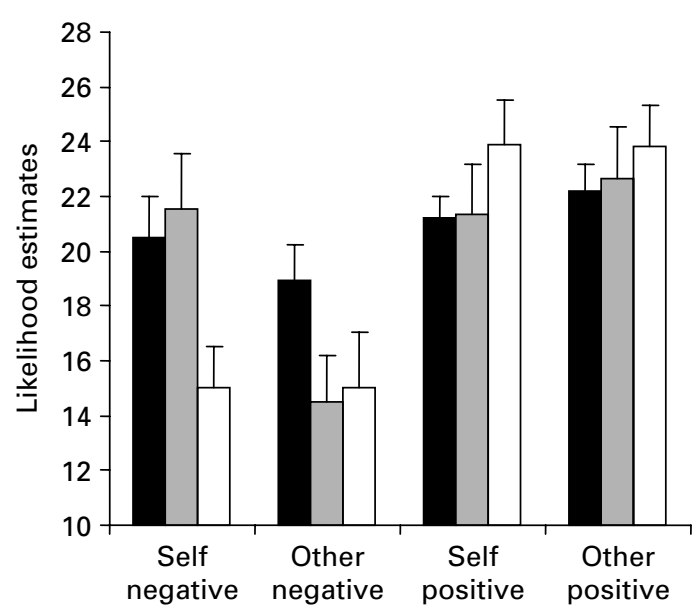

Fig. 1. Summed likelihood estimates for negative and positive events, across self and other, for the seasonal depression ( $\square$ ), control $(\square)$ and non-seasonal depression ( $\square$ ) groups. Values are means, with standard errors represented by vertical bars.

completed the latter two measures only. Participants were assessed in the winter in the UK at a time of year with an average of $8.75 \mathrm{~h}$ of daylight and a mean temperature of $6^{\circ} \mathrm{C}$.

\section{Results}

Table 1 presents the descriptive data for the three groups. The groups did not differ significantly in terms of gender ratio $\left(\chi^{2}=3.00, \mathrm{df}=2, p>0.05\right)$ or age $[F(2,59)=2.95, p=0.06]$, although there was an overall trend for age that was accounted for by the NSD group tending to be older than the SD group ( $p=0.06$, posthoc Scheffé test). Consequently, all key analyses were repeated with age as a covariate. The patterns of findings were unaltered and so the results without age entered as a covariate are reported below.

As expected, there were significant differences across groups on current self-reported depression and anxiety (all $F^{\prime}$ s $>13.76$, all $p^{\prime}$ s $<0.001$ ), with the NSD and SD groups scoring higher than controls on post-hoc tests (all $p^{\prime} \mathrm{s}<0.001$ ), but not significantly differently to each other (all $p^{\prime}$ s $>0.05$ ). Mean SIGH-SAD scores for the SD group are comparable with other studies (Rohan et al. 2007), attesting to the representativeness of the SD sample.

\section{LEM performance}

Scores for each participant for self-related negative, self-related positive, other-related negative and otherrelated positive events on the LEM were calculated by summing the separate probability estimates for the six items in each event category (see Fig. 1). 
A group (SD, NSD, controls) $\times$ valence (negative, positive) $\times$ reference (self, other) mixed-model analysis of variance (ANOVA) examined the study hypotheses. If the profile of likelihood estimations for negative (but not positive) events across self and other differed significantly across the groups as anticipated, then one would expect a significant three-way interaction of reference $\times$ valence $\times$ group.

The results revealed a significant main effect of valence $\left[F(1,59)=21.24, p<0.001, \eta_{p}^{2}=0.27\right]$, with positive events being rated as more likely than negative events. This is in line with previous findings (Butler \& Mathews, 1983) and probably reflects differences in the objective likelihood of the respective events. As it is not central to the present hypotheses it will not be discussed further. There were also significant interactions of reference $\times$ valence $[F(1,59)=9.96, p<0.001$, $\left.\eta_{p}^{2}=0.14\right]$ and group $\times$ valence $[F(2,59)=3.91, p<0.05$, $\left.\eta_{p}^{2}=0.12\right]$, which were qualified by the predicted significant interaction of reference $\times$ valence $\times$ group $\left[F(2,59)=3.87, p<0.05, \eta_{p}^{2}=0.12\right]$. None of the other main effects or interactions was significant.

This three-way interaction of reference $\times$ valence $x$ group was deconstructed by looking at estimations for positive and negative events separately. For positive events, in line with the previous literature (Butler \& Mathews, 1983), there were no significant main effects or interactions. In contrast, for negative events there was a main effect of reference $[F(1,59)=12.32, p<$ $\left.0.001, \eta_{p}^{2}=0.17\right]$, but no main effect of group $[F(2,59)=$ 2.36, $p=0.10]$. These effects were qualified by the predicted group $\times$ reference interaction $[F(2,59)=5.69$, $\left.p<0.01, \eta_{p}^{2}=0.16\right]$.

To deconstruct this interaction and to address our specific hypotheses we examined the reference effect for negative events for each group separately. As expected, for both the SD $[t(32)=1.32, p=0.20$, Cohen's $d=0.20]$ and control participants $[t(14)<1]$, there were no significant effects of reference. However, there was the predicted relative elevation in estimations for self-referent events in the NSD group $[t(13)=5.84, p<0.01$, Cohen's $d=1.03]$.

To verify that this self-referent bias for negative events in the NSD group differed significantly from the flatter profiles for both the SD and control groups, we conducted two further group (NSD $v$. SD; NSD $v$. control) $\times$ reference (self $v$. other) ANOVAs. In both cases the group $\times$ reference interaction term was significant as expected: NSD $v$. SD $[F(1,45)=7.17$, $\left.p<0.02, \eta_{p}^{2}=0.14\right]$; NSD $v$. controls $[F(1,27)=17.31$, $\left.p<0.01, \eta_{p}^{2}=0.39\right]$. In contrast, a similar group (SD $v$. control) $\times$ reference (self $v$. other) ANOVA for negative events revealed no such significant group $\times$ reference interaction for the control and SD groups $(F<1)$, although the SD group estimated negative events as more likely to happen overall than did controls $\left[F(1,46)=4.57, p<0.05, \eta_{p}^{2}=0.09\right]$.

Examination of Fig. 1 indicates that the significant reference $\times$ group interaction for negative event estimations across SD and NSD described above may be a function of the two groups being comparable for selfrelated events but different for other-related events. We examined this statistically by breaking down this interaction by considering self and other separately. While there was no significant difference for selfreferent events $(t<1)$, the SD group did indeed estimate other-referent events as more likely to happen than did the NSD group $[t(45)=2.09, p<0.05$, Cohen's $d=0.62]$.

\section{Discussion}

The present study examined the cognitive processing of future-related self-referent emotional information in SD and NSD for the first time. As predicted, NSD participants estimated negative events as more likely to happen to themselves than to others (a negative selfreferent bias), replicating previous findings (Butler \& Mathews, 1983), whereas there was no support for such a self-reference effect in SD participants or in never-depressed controls. Instead, SD participants estimated that negative events would be more likely to happen to both themselves and other people, relative to the estimates of the controls, and more likely to happen to others compared with the estimates of the NSD group. As anticipated there were no group differences in estimations for positive events in line with previous findings (Butler \& Mathews, 1983).

These data provide further evidence, this time in the domain of future event processing, that biases for negative self-referent emotional information in SD exhibit a different pattern from that in NSD. To our knowledge the findings also represent the first demonstration of any kind of differential cognitive processing across SD and NDS, assessed in the same study.

It is notable that the lack of a self-reference bias in SD patients was because they produced elevated likelihood estimations for negative events for both self and other, whereas in NSD there was only a relative elevation for self-referred negative events. This appears not to be a function of overall levels of symptomatology as the SD group actually scored lower on average (albeit non-significantly) on the self-report measures of depression and trait and state anxiety that all participants completed than did the NSD group. A possible explanation for the effect is that likelihood estimations in SD represent a global negative bias potentially as a function of depressed mood itself, whereas in NSD they reflect the presence of 
underlying negative self-referent mental schemas (Dalgleish et al. 2001, 2004).

It is important to continue to build an understanding of how negative information about the self and the world is processed in SD in the context of the rapid development of cognitive-behavioural interventions for the condition that draw upon knowledge of the cognitive profile associated with the syndrome (Rohan et al. 2007). The present data, allied to the previous findings on memory biases (Dalgleish et al. 2001, 2004), suggest that the self-referential nature of cognitive processing may be an important theme in this endeavour.

A limitation of the present study was that the sample sizes in the NSD and never-depressed control groups were relatively modest. However, in no instance did a predicted effect fail to reach significance due to lack of power and the non-significant findings all had small to trivial effect sizes, suggesting that sample size did not limit the results. Furthermore, the present sample sizes were comparable with those in previous similar studies.

\section{Acknowledgements}

We thank Helen Spinks for help with the data collection. This work was funded by the UK Medical Research Council (U.1055.02.002.00001.01).

\section{Declaration of Interest}

None.

\section{References}

Andersen SM, Spielman LA, Bargh JA (1992). Future-event schemas and certainty about the future: automaticity in depressives' future-event predictions. Journal of Personality and Social Psychology 63, 711-723.

APA (1994). Diagnostic and Statistical Manual of Mental Disorders, 4th edn, revised. American Psychiatric Association: Washington, DC.

Beck AT (1967). Depression: Clinical, Experimental and Theoretical Aspects. Harper \& Row: New York.

Beck AT (2005). The current state of cognitive therapy - a 40-year retrospective. Archives of General Psychiatry 62, 953-959.

Beck AT, Rush AJ, Shaw BF, Emery G (1979). Cognitive Therapy of Depression: A Treatment Manual. Guilford Press: New York.

Beck AT, Ward CH, Mendelson M, Mock J, Erbaugh J (1961). An inventory for measuring depression. Archives of General Psychiatry 4, 53-63.

Bisson MAS, Sears CR (2007). The effect of depressed mood on the interpretation of ambiguity, with and without negative mood induction. Cognition and Emotion 21, 614-645.
Blaney PH (1986). Affect and memory: a review. Psychological Bulletin 99, 229-246.

Butler G, Mathews A (1983). Cognitive processes in anxiety. Advances in Behavioural Research and Therapy 5, 51-62.

Dalgleish T, Spinks H, Golden AM, du Toit P (2004). Processing of emotional information in seasonal depression across different cognitive measures. Journal of Abnormal Psychology 113, 116-126.

Dalgleish T, Spinks H, Yiend J, Kuyken W (2001). Autobiographical memory style in seasonal affective disorder and its relationship to future symptom remission. Journal of Abnormal Psychology 110, 335-340.

First MB, Spitzer RL, Gibbon M, Williams JBW (1997). Structured Clinical Interview for DSM-IV. American Psychiatric Press: Washington, DC.

Golden AM, Dalgleish T, Spinks H (2006). Dysfunctional attitudes in seasonal affective disorder. Behaviour Research and Therapy 44, 1159-1164.

Gregory WL, Cialdini RB, Carpenter KM (1982). Selfrelevant scenarios as mediators of likelihood estimates and compliance: does imagining make it so. Journal of Personality and Social Psychology 43, 89-99.

Hammen C, Krantz S, Cochran S (1981). Relationships between depression and causal attributions about stressful life events. Cognitive Therapy and Research 5, 351-358.

Hodges S, Marks M (1998). Cognitive characteristics of seasonal affective disorder: a preliminary investigation. Journal of Affective Disorders 50, 59-64.

Hollon SD, Kendall PC (1980). Cognitive self-statements in depression: development of an automatic thoughts questionnaire. Cognitive Therapy and Research 4, 383-395.

Levitan RD, Rector NA, Bagby RM (1998). Negative attributional style in seasonal and nonseasonal depression. American Journal of Psychiatry 155, 428-430.

Mathews A, Ridgeway V, Williamson DA (1996). Evidence for attention to threatening stimuli in depression. Behavior Research and Therapy 34, 695-705.

Michalon M, Eskes GA, Mate-Kole CC (1997). Effects of light therapy on neuropsychological function and mood in seasonal affective disorder. Journal of Psychiatry and Neuroscience 22, 19-28.

O'Brien JT, Sahakian BJ, Checkley SA (1993). Cognitive impairments in patients with seasonal affective disorder. British Journal of Psychiatry 163, 338-343.

Reich DA, Weary G (1998). Depressives' future-event schemas and the social inference process. Journal of Personality and Social Psychology 74, 1133-1145.

Rohan KJ, Roecklein KA, Lindsey KT, Johnson LG, Lippy RD, Lacy TJ, Barton FB (2007). A randomized controlled trial of cognitive-behavioral therapy, light therapy, and their combination for seasonal affective disorder. Journal of Consulting and Clinical Psychology 75, 489-500.

Rohan KJ, Sigmon ST, Dorhofer DM (2003). Cognitive-behavioral factors in seasonal affective disorder. Journal of Consulting and Clinical Psychology 71, 22-30.

Rosenthal NE, Sack DA, Gillin JC, Lewy AJ, Goodwin FK, Davenport Y, Mueller PS, Newsome DA, Wehr TA (1984) Seasonal affective disorder: a description of the syndrome and preliminary findings with light therapy. Archives of General Psychiatry 41, 72-80. 
Rosenthal NE, Wehr TA (1992). Towards understanding the mechanism of action of light in seasonal affective disorder. Pharmacopsychiatry 25, 56-60.

Sohn CH, Lam RW (2005). Update on the biology of seasonal affective disorder. CNS Spectrums 10, 635-646.

Spielberger CD, Gorsuch RL, Lushene R (1970). Manual for the State-Trait Anxiety Inventory. Consulting Psychologists Press: Palo Alto, CA.

Spinks H, Dalgleish T (2001). Attentional processing and levels of symptomatology in SAD: a preliminary study. Journal of Affective Disorders 62, 229-232.

Sullivan B, Payne TW (2007). Affective disorders and cognitive failures: a comparison of seasonal and nonseasonal depression. American Journal of Psychiatry 164, 1663-1667.

Weniger G, Lange C, Rüther E, Irle E (2004). Differential impairments of facial affect recognition in schizophrenia subtypes and major depression. Psychiatry Research $\mathbf{1 2 8}$ 135-146.

Williams JB, Link MJ, Rosenthal NE, Amira L, Terman M (1988). Structured Interview Guide for the Hamilton Depression Rating Scale - Seasonal Affective Disorder Version. Society for Light Treatment and Biological Rhythms: Wilsonville, OR.

Williams JMG, Barnhofer T, Crane C, Hermans D, Raes F, Watkins E, Dalgleish T (2007). Autobiographical memory specificity and emotional disorder. Psychological Bulletin 133, 122-148.

Williams JMG, Watts FN, MacLeod C, Mathews A (1997). Cognitive Psychology and Emotional Disorders, 2nd edn. John Wiley and Sons: Chichester, UK.

Young MA, Azam OA (2003). Ruminative response style and the severity of seasonal affective disorder. Cognitive Therapy and Research 27, 223-232. 